\title{
Perfil de las Publicaciones sobre Bienestar Subjetivo en Chile
}

\author{
Subjective Wellbeing publications in Chile
}

Mg. Fernando Farías Olavarría (ffarias@ubiobio.cl) Facultad de Educación y Humanidades, Universidad del Bío-Bío (Concepción, Chile)

Mg. Cristian Orellana Fonseca (corellana@ubiobio.cl) Facultad de Educación y Humanidades, Universidad del Bío-Bío (Concepción, Chile)

Dra. Claudia Pérez (cperezs@udec.cl) Departamento de Psicología, Universidad de Concepción (Concepción, Chile)

\begin{abstract}
This article aims to carry out an analysis of the publications about subjective wellbeing that have been developed in Chile. To reach such an objective, all the publications indexed in the main databases were gathered. The analysed variables were: type of research according to its thoroughness, epistemological stance, disciplinary areas of the researchers and characteristics of the journal. The data were analysed through univariate descriptive statistics and analysis of multiple correspondences. The main results indicate that the first publications start in the decade of 2000, and are concentrated in the disciplinary field of psychology, basically using a quantitative research methodology, with correlational analyses, transversal, non-experimental and macro-subjective levels.
\end{abstract}

Key words: subjectivity, wellbeing, publications, bibliometric analysis, research.

\section{Resumen}

En el presente artículo se realiza un análisis de las publicaciones sobre el bienestar subjetivo desarrolladas en Chile. Para hacerlo se acopió el total de publicaciones indexadas en las principales bases de datos. Las variables analizadas fueron: tipo de investigación según su profundidad, enfoques epistemológicos, áreas disciplinarias de los investigadores y características de las revistas. Los datos se analizaron a través de estadística descriptiva univariada y análisis de correspondencias múltiples. Los principales resultados indican que las primeras publicaciones se inician a partir de la década del 2000, se concentran en el campo disciplinario de la psicología, fundamentalmente con enfoque cuantitativo, los análisis son de tipo correlacional, transversal, no experimental y de niveles macro-subjetivos.

Palabras clave: bienestar, subjetividad, publicaciones, análisis bibliométrico, investigación.

\section{Introducción}

La felicidad ha sido una constante preocupación en el pensamiento, en sus inicios asentado en el mundo religioso, luego en el filosófico, pero será desde mediados del siglo XX cuando pasa a ser objeto de 
estudio en el campo de la ciencia. Su interés ha estado en la comprensión, medición y explicación de los factores que subyacen al concepto. De este modo, los primeros estudios empíricos en esta línea datan de 1967, específicamente con W. Wilson, quien construye un perfil de las características psicosociales que poseen las personas felices en los Estados Unidos, siendo éste joven, de cualquier sexo, saludable, inteligente, educado, extrovertido, optimista, libre, religioso, con altos niveles de autoestima, de aspiraciones modestas y con una moral del trabajo (Diener et al. 1999).

Este fenómeno de estudio se ha abordado desde diferentes conceptualizaciones, tales como bienestar emocional o psicológico, felicidad, satisfacción con la vida, afecto positivo, entre otros. Las diferencias entre éstas se dan en virtud de los énfasis, así como de los abordajes disciplinarios de los investigadores. De este modo el concepto de bienestar emocional o psicológico es comprendido como el resultado de cambios socioculturales en la salud mental de los individuos, como también, percepción de la salud física, interés por la vida, actitudes positivas, entre otras (Pinquart y Silbereisen 2004). También se ha pensado como bienestar psicosocial; a nuestro entender una concepción de mayor complejidad en tanto incorpora tanto el bienestar emocional o psicológico, así como el bienestar social y colectivo.

De acuerdo con Martikainen, Bartley y Lahelma (2002) el concepto psicosocial se vincula con el nivel meso-social de la teoría ecológica de Bronfenbrenner. Bajo la arquitectura teórica de Bronfenbrenner se identifican cuatro niveles de sistemas: a) microsistema: el cual incluye a los sujetos mismos, con sus características personales de carga genética, edad, género y el entorno social inmediato en el que participa, donde mantiene relaciones próximas, cara a cara (familia, escuela, grupos, etc.); b) mesosistema: el sistema formado por las interacciones de los microsistemas, de los distintos contextos en que los sujetos participan (por ejemplo, relaciones comunitarias); c) exosistema: el medio social externo a los sujetos, en el que no participa en forma directa pero que afecta los entornos en que él participa (por ejemplo, el entorno laboral de sus padres, los servicios locales de salud); y d) macrosistema: los sistemas institucionales, sociales y culturales o subculturales que enmarcan los otros sistemas en que los sujetos participan (ideologías, valores, prácticas, leyes, políticas públicas, medios de comunicación, sociedad global, entre otros).

Otros de los términos utilizados para la aprehensión de la felicidad ha sido el de calidad de vida, que en cierto sentido es equivalente al de bienestar psicosocial, en tanto incluye las dimensiones emocionales, sociales y físicas. Sin embargo, este concepto ha sido utilizado de manera restringida en el campo de la investigación médica en tanto lo limita al impacto que puede tener una enfermedad en el bienestar de los individuos. Desde la psicología se han acuñado diferentes conceptos los cuales pretenden dar cuenta de éste fenómeno, con sus respectivos matices conceptuales, así encontramos bienestar emocional o psicológico, el cual orienta su atención a los procesos y logro de aquellos valores que hacen sentir vivos a las personas. El modelo que más se ha utilizado en este sentido ha sido el de Carol Ryff, el cual se estructura a partir de seis dimensiones del bienestar: auto-aceptación, relaciones positivas, dominio del entorno, autonomía, propósitos en la vida y crecimiento personal (Ryff 1989, Ryff y Keyes 1995).

Otro concepto es el de afecto positivo, el cual crea una escala que mide las experiencias emocionales de las personas, tanto en sus emociones positivas (alegría, satisfacción, placer, orgullo, cariño, felicidad, euforia) y negativas (miedo, ira, tristeza, enfado, estrés, culpa y vergüenza, depresión, envidia). Los individuos con mayores niveles de satisfacción es el producto de la diferencia entre sus sentimientos positivos con los negativos, y en donde predominan los afectos positivos por sobre los negativos. También encontramos el concepto de satisfacción con la vida, el cual es entendido como una evaluación global que realizan los sujetos acerca de su vida en alusión a un umbral de comparación. En el mismo 
concepto, también se utiliza la satisfacción en diferentes dominio de la vida, tales como trabajo, salud, familia, tiempo libre, entre otros. Otro de los términos utilizado es bienestar subjetivo, el cual concentra nuestra atención y es entendido como "una amplia categoría de fenómenos que incluye las respuestas emocionales de las personas, la satisfacción con los dominios, y los juicios globales sobre la satisfacción con la vida" (Diener et.al. 1999: 277). La definición del concepto contiene dos componentes: cognitivo y afectivo. Tales dimensiones se vinculan con espacios temporales diferentes, así, satisfacción con la vida (cognitivamente) se corresponde con un marco temporal de largo plazo, en tanto un juicio acerca de la vida de la persona; en cambio lo afectivo se relaciona con una dimensión temporal de lo inmediato, dado que se corresponde con un juicio de balance de los afectos positivos y negativos que está experimentando el individuo.

Los estudios del bienestar subjetivo han sido desarrollados fundamentalmente por el área disciplinaria de la psicología, en donde el grado de importancia que ha adquirido al interior de ésta en las últimas décadas ha llevado a reconocer la presencia de una nueva sub-área dentro de esta disciplina, denominada psicología positiva. A nuestro juicio, es posible reconocer la importancia de la investigación empírica y teórica al interior de la psicología, pero es de justicia indicar que ésta temática también ha estado presente en otras áreas disciplinarias de las ciencias sociales como ha sido el caso de la economía, cuya relevancia teórica se encuentra presente hasta hoy tras los aportes de J. Bentham. En otros campos disciplinarios como la ciencia política, sociología y trabajo social también ha estado presente, fundamentalmente con acciones tendientes a generar mayores niveles de bienestar y de calidad de vida en los miembros de la sociedad. Será específicamente bajo el concepto de calidad de vida donde entra el componente de bienestar subjetivo o satisfacción con la vida que es abordado por estos campos disciplinarios. Además de la condiciones de materialidad u objetivas necesarias para una "buena vida", entra el componente de cómo perciben la práctica de la vida los sujetos o el componente subjetivo de la calidad de vida.

La importancia que ha adquirido en los últimos años el estudio del bienestar subjetivo en el mundo se aprecia en la incorporación de esta temática en múltiples organismos interestatales, así el año 2008 el gobierno francés genera una comisión de análisis que elabora el "Informe de la Comisión sobre la Medición del Desarrollo Económico y del Progreso Social”, como también la resolución 65/309 de Naciones Unidas de 19 de julio de 2011, en donde insta a las naciones a que emprendan nuevas medidas que reflejen la importancia de la búsqueda de la felicidad y el bienestar en el desarrollo con miras a orientar sus políticas públicas. De igual modo la Organización de Cooperación y Desarrollo Económico (OCDE), el año 2011, lanza el índice para una Vida Mejor (Your Better Life Index); medida compuesta por once dimensiones (vivienda, ingresos, empleo, comunidad, educación, medio ambiente, compromiso cívico, salud, satisfacción ante la vida, seguridad y balance vida-trabajo), que son medidas para sus 36 naciones miembros. También es posible observar la importancia que tiene este campo de estudio a través de múltiples fundaciones y centros, así como a la intervención bajo esta perspectiva. A modo de ejemplo, el Instituto Coca-Cola de la felicidad en España, el Centre for Well-being en Inglaterra que genera el Happy Planet Index; en los Países Bajos el World Database of Happiness desarrollado por Ruth Veenhoven; la Encuesta Mundial de Valores y en Latinoamérica a través de las investigaciones y reportes del Latinobarómetro.

El bienestar subjetivo como objeto de estudio en el campo de las ciencias sociales en Chile es de data reciente, al menos así se evidencia en la investigación empírica del país, a pesar que la temática se viene desarrollando a nivel mundial desde los años setenta. En los estudios chilenos, el interés por este objeto 
ha sido desarrollado principalmente desde el campo disciplinario de la psicología y a partir de los últimos años, ha comenzado a ser estudiado empíricamente desde diferentes áreas disciplinarias.

En el campo de las políticas públicas, han sido diferentes países del mundo que han incorporado el bienestar subjetivo como prioritario en sus naciones, de este modo el Reino de Bután en los años setenta crea el Ministerio de la Felicidad y comienza a innovar por medio de la medición de la Felicidad Nacional Bruta. En el contexto latinoamericano Bolivia, Ecuador, Venezuela e incipientemente Chile han generado desde el Estado políticas orientadas a incrementar la satisfacción con la vida de sus ciudadanos. En el caso de Chile, esta tendencia se puede observar a través del estudio solicitado por el gobierno al Programa de Naciones Unidas para el Desarrollo (PNUD 2012) sobre el estado del Bienestar Subjetivo en Chile. Lo relevante de éste estudio es la perspectiva del PNUD, en cuanto propone un modelo integral para la comprensión del bienestar subjetivo, al considerar tanto su dimensión individual (yo ensimismado) y social (yo en sociedad), los cuales se relacionan de manera compleja y se combinan de diferente modos. Esta tendencia se observa de igual modo en la incorporación de la pregunta sobre la satisfacción con la vida en la encuesta CASEN 2011. Finalmente y de modo ilustrativo se puede indicar la inclusión de éste tópico en algunos programas gubernamentales, como han sido "Elige vivir Sano" y "Vejez Positiva".

Para la comprensión de las investigaciones desarrolladas en Chile, es posible hacer uso de la propuesta metateórica desarrollada por el sociólogo Georg Ritzer (1997), el cual hace uso de dos dimensiones. a) Un eje $X$ da cuenta del continuo objetivo-subjetivo. De este modo, los fenómenos objetivos se refieren a aquellos que poseen existencia material, como por ejemplo, los actores sociales, la interacción, las instituciones sociales, los ingresos económicos, la familia y sus tipologías, entre otros; en cambio en el extremo subjetivo estarían aquellos fenómenos orientados a dar cuenta de los procesos de construcción social de la realidad, las normas, valores, cultura, entre otros. b) El eje $Y$ da cuenta del continuo que transita entre los polos macro-micro, donde los fenómenos macro son aquellos que involucran sistemas sociales, nacionales, regionales y/o comunales; en su extremo micro se encuentra la conducta y/o acción individual.

Como se ha indicado, no son relaciones dicotómicas entre los extremos, sino un continuo en cada uno de estos posicionamientos. A modo de complementar la propuesta del plano metateórico de Ritzer, es posible incluir un tercer eje que dé cuenta de la temporalidad del fenómeno investigado, en tanto, los objetos de estudios no se limitan exclusivamente a diseños transversales. También es posible estudiar procesos o el cambio a través de diseños longitudinales. Cabe indicar que los diseños longitudinales pueden ser planificados a partir de su temporalidad de manera retrospectiva o prospectivamente.

A modo de ejemplo, en los diseños longitudinales retrospectivos, nos encontraríamos en aquellas investigaciones que, utilizando datos secundarios, informan sobre la evolución o cambio en el tiempo, como han sido las investigaciones que haciendo uso de los datos censales disponibles estudian las variaciones de algún fenómeno, o bien, bajo un enfoque cualitativo la representación social de la felicidad en diferentes momentos históricos. En cambio, en una investigación prospectiva el interés se encuentra en observar la variación tras intervenciones clínicas o psicosociales y cómo éstas se mantienen en el tiempo.

Finalmente, es necesario indicar que todo objeto de estudio es una construcción que realiza la ciencia para comprender o explicar fenómenos. De este modo el estudio del bienestar subjetivo es un objeto construido que puede ser investigado desde diferentes dimensiones del plano que nos propone Ritzer, a 
modo de ilustración, en la dimensión naturaleza del objeto en su componente subjetivo es cuando el investigador está interesado en el proceso de construcción de los significados que atribuyen los sujetos al bienestar subjetivo; en cambio, en su opuesto objetivo, el interés de los investigadores está en observar factores que influyen o determinan los niveles de bienestar, como ha sido los estudios sobre la relación y dependencia entre satisfacción con la vida y estructura cultural de la sociedad (individualistas vs colectivistas). En cambio en la dimensión nivel de análisis del objeto, el componente microsocial se observa cuando el investigador está centrado en reconocer el conjunto de elementos que determinan en su conciencia el juicio sobre el bienestar subjetivo, y lo macrosocial se presenta en las mediciones que actualmente se desarrollan, como es el caso del Reino de Bután que mide la felicidad interna bruta, o la OCDE con el Índice para una Vida Mejor (Your Better Life Index).

\section{Material y método}

Se realizó un acopio de los artículos publicados en Chile a partir de las bases de datos Redalyc, Dialnet, Scielo, Scopus y WoS Thompson Reuter, donde los criterios de inclusión de los artículos fueron: que dentro de las palabras claves se encuentre "bienestar subjetivo", "bienestar psicológico" o "satisfacción con la vida" y que hayan sido publicados en revistas chilenas y/o por investigadores chilenos. Se construyó una base de datos con un total de 48 artículos entre los años 2002 y 2013. Las variables consideradas son: título de los artículos, tipo de investigación según criterios de M. Cea (2001), para la cual las investigaciones pueden ser agrupadas de acuerdo a los objetivos del estudio en exploratorio, descriptivo, correlacional, causal, evaluativo y teórico; número de autores, formación disciplinar del autor principal, orientación epistemológica, cuyas categorías utilizadas fueron las principales en el campo de las ciencias sociales, tales como enfoque empírico-analítico, crítico-dialéctico y sociofenomenológico o hermenéutico; nombre de la revista, indexación de la revista (cuando éstas se encuentran indexadas en dos o más se clasifica en la de mayor nivel de prestigio en el ámbito académico); clasificación de los artículos en función de la propuesta metateórica de G. Ritzer (1997) que como se ha indicado anteriormente, pueden estar dentro del continuo objetivo-subjetivo y micro-macro; además, dimensión temporal de los estudios, que fueron clasificado en longitudinales o transversales.

Los análisis realizados fueron de carácter descriptivo univariados con la finalidad de describir las características principales de los artículos publicados durante el periodo de estudio, y de correspondencias múltiples, con el propósito de explorar agrupamientos y relaciones entre las variables y representarlas gráficamente en el plano factorial.

\section{Análisis y resultados}

Uno de los indicadores más sencillos para observar el desarrollo de un campo disciplinario es el número de publicaciones. En la tabla 1 se observa la evolución y desenvolvimiento de este campo de estudio, pudiéndose apreciar que es de data reciente en Chile en comparación del desarrollo a nivel internacional, el cual se viene dando desde la década de 1970 (con el primer estudio empírico de Wilson) y con fuerza a partir de la década de 1990 y 2000. 
Tabla 1. Índice de colaboración anual

\begin{tabular}{|l|r|r|r|r|c|}
\hline \multirow{2}{*}{ Año } & \multicolumn{2}{|c|}{$\begin{array}{c}\text { Trabajos } \\
\text { Originales }\end{array}$} & \multicolumn{2}{|c|}{ Autores } & \multirow{2}{*}{$\begin{array}{c}\text { Índice } \\
\text { Colaboración }\end{array}$} \\
\cline { 2 - 5 } & \multicolumn{1}{|c|}{$\mathrm{n}$} & \multicolumn{1}{c|}{$\%$} & \multicolumn{1}{c|}{$\mathrm{n}$} & \multicolumn{1}{c|}{$\%$} & \\
\hline 2002 & 1 & $2,2 \%$ & 2 & $2,2 \%$ & 2,0 \\
\hline 2003 & 1 & $2,2 \%$ & 2 & $2,2 \%$ & 2,0 \\
\hline 2004 & 1 & $2,2 \%$ & 6 & $2,2 \%$ & 6,0 \\
\hline 2006 & 1 & $2,2 \%$ & 1 & $2,2 \%$ & 1,0 \\
\hline 2007 & 3 & $6,7 \%$ & 13 & $6,7 \%$ & 4,3 \\
\hline 2008 & 6 & $13,3 \%$ & 14 & $13,3 \%$ & 2,3 \\
\hline 2009 & 2 & $4,4 \%$ & 4 & $4,4 \%$ & 2,0 \\
\hline 2010 & 4 & $8,9 \%$ & 13 & $8,9 \%$ & 3,3 \\
\hline 2011 & 10 & $22,2 \%$ & 30 & $22,2 \%$ & 3,0 \\
\hline 2012 & 4 & $8,9 \%$ & 8 & $8,9 \%$ & 2,0 \\
\hline 2013 & 12 & $26,7 \%$ & 36 & $26,7 \%$ & 3,0 \\
\hline TOTAL & 45 & $100,0 \%$ & 129 & $\mathbf{1 0 0 , 0 \%}$ & $\mathbf{2 , 8}$ \\
\hline
\end{tabular}

Fuente: Elaboración propia

De igual modo, la tabla 1 nos muestra la evolución en el número de publicaciones que se vienen desarrollando en el país durante los últimos once años, en donde el promedio de artículos es de 4,1 y su desviación típica de 3,8 por año. El índice de colaboración de las publicaciones en el conjunto de los once años analizados es de 2,8 (tabla 1). El valor de este índice, de acuerdo con R. Sancho (1990), se encuentra dentro del promedio de colaboración de los diferentes campos disciplinarios.

\section{Tabla 2. Campo disciplinario de autor principal}

\begin{tabular}{|l|r|r|}
\hline Disciplina & \multicolumn{1}{|c|}{$\mathbf{n}$} & \multicolumn{1}{c|}{$\%$} \\
\hline Psicología & 33 & $68,8 \%$ \\
\hline Economía & 6 & $12,5 \%$ \\
\hline Agricultura & 2 & $4,2 \%$ \\
\hline Psiquiatría & 2 & $4,2 \%$ \\
\hline Sociología & 2 & $4,2 \%$ \\
\hline Antropología & 1 & $2,1 \%$ \\
\hline Enfermería & 1 & $2,1 \%$ \\
\hline Terapeuta ocupacional & 1 & $2,1 \%$ \\
\hline TOTAL & $\mathbf{4 8}$ & $\mathbf{1 0 0 \%}$ \\
\hline
\end{tabular}

Fuente: Elaboración propia

En la tabla 2, y en concordancia con los estudios internacionales, ha sido la psicología (68,8\%) la disciplina que contribuye mayoritariamente al desarrollo de este campo. El aporte ha sido de tal magnitud que actualmente se habla al interior de este campo disciplinario de un nueva sub-área disciplinaria: la psicología positiva. Le sigue, pero en un nivel mucho más bajo, la economía (12,5\%), siendo esta disciplina una de las que más contribuciones ha realizado al estudio del bienestar. Como ejemplo emblemático basta recordar la contribución realizada por J. Bentham presente hasta el día de hoy en las investigaciones sobre el bienestar subjetivo, en lo relativo a la perspectiva hedónica. 
Tabla 3. Tipo de investigación

\begin{tabular}{|l|c|c|}
\hline Investigación & $\mathbf{n}$ & $\mathbf{\%}$ \\
\hline Descriptivo & 3 & $6,7 \%$ \\
\hline Correlacional & 24 & $53,3 \%$ \\
\hline Causal & 8 & $17,8 \%$ \\
\hline Evaluativo & 2 & $4,4 \%$ \\
\hline Teórica & 8 & $17,8 \%$ \\
\hline Total & $\mathbf{4 5}$ & $\mathbf{1 0 0 , 0} \%$ \\
\hline
\end{tabular}

Fuente: Elaboración propia

La tabla 3 nos muestra el tipo de estudio que se vienen desarrollando en Chile en función de la profundidad de los objetivos del estudio, de acuerdo a lo que sostiene M. Cea (2001), en donde predominan los estudios correlacionales cuyas variables de asociación con el bienestar subjetivo fueron principalmente características sociodemográficas; en cambio para los estudios causales las variables predictoras fueron condiciones laborales y factores salutogénicos. Las investigaciones evaluativas se orientaron a validación de escalas de bienestar subjetivo en médicos y personas con cuadros de esquizofrenia. Las publicaciones teóricas se orientaron a discutir la pertinencia así como la evolución del concepto de bienestar subjetivo (revisiones sistemáticas). Finalmente, los estudios descriptivos midieron los niveles de bienestar subjetivo en grupos etarios específicos tales como jóvenes y adultos mayores, junto a variables sociodemográficas.

En la tabla 4 se presenta la indexación de las revistas donde publican los investigadores que cultivan dicho campo. Se observa el interés de éstos en comunicar sus resultados en aquellas revistas indexadas en Thompson Reuter (WoS), Scielo y Scopus las cuales concentran el 87,6\%.

\section{Tabla 4. Indexación de las revistas donde publican los artículos}

\begin{tabular}{|l|c|c|}
\hline Indexación & $\mathbf{n}$ & $\mathbf{\%}$ \\
\hline ISI & 19 & $42,2 \%$ \\
\hline Scielo & 5 & $11,1 \%$ \\
\hline Scopus & 15 & $33,3 \%$ \\
\hline Dialnet & 3 & $6,7 \%$ \\
\hline Latindex & 2 & $4,4 \%$ \\
\hline Ebsco & 1 & $2,2 \%$ \\
\hline Total & $\mathbf{4 5}$ & $\mathbf{1 0 0 , 0 \%}$ \\
\hline
\end{tabular}

Fuente: Elaboración propia

Del conjunto de artículos publicados en revistas indexadas, en Thompson Reuters (WoS) 14 han sido publicadas en revistas chilenas: Terapia Psicológica (6), Revista Médica de Chile (4), Magallania (3) y Teología y Vida (1). 3 artículos en revistas españolas, 2 en brasileñas y colombianas. En relación con los artículos publicados en revistas indexadas en SciELO, 14 corresponden a revistas chilenas (93,3\%), las cuales no presentan moda, dada la heterogeneidad de revistas donde son publicados tales artículos. Solo un artículo $(6,6 \%)$ fue publicado en una revista argentina. Finalmente, de las publicaciones en revistas Scopus, 3 corresponden a revistas chilenas ( 2 en Universum y 1 en Psyke); los restantes 3 artículos son de revistas procedentes de Argentina, Colombia y Brasil. 
En lo relativo al marco epistemológico en el cual se inscriben los artículos, en este trabajo se tuvo en consideración los tres principales programas de investigación en el campo de las ciencias sociales: empírico-analítico, crítico-dialéctico y sociofenomenológico. Al clasificar los artículos bajo este criterio, encontramos 39 (81,3\%) publicaciones adscrita al programa empírico-analítico, $9(18,8 \%)$ en el sociofenomenológico y ausencia total de artículos bajo el programa crítico-dialéctico.

Como se ha indicado anteriormente, el objeto de estudio puede ser observado a través de la propuesta de metateoría desarrollada por G. Ritzer (1997), donde los continuo del eje X transitan desde fenómenos de estudios objetivos-subjetivo, en cambio el continuo representado por el $Y$ circula desde fenómenos macro-micro. Al aplicar esta perspectiva a los estudios del bienestar subjetivo, encontramos 41 artículos $(91,1 \%)$ en el espacio objetivo y 4 artículos $(8,9 \%)$ en el espacio subjetivo. En cambio en el eje $Y$, se observan 29 artículos (64,4\%) circunscritos en el espacio microsocial y 16 publicaciones (35,6\%) se clasifican en el espacio macrosocial. La dimensión temporal (eje Z), la cual da cuenta del continuo temporal o longitudinal (tanto en una perspectiva prospectivos o retrospectivos), encontramos que la totalidad de los estudios del bienestar subjetivo realizados en Chile se inscriben en una temporalidad transversal.

Para establecer el perfil de las publicaciones del bienestar subjetivo en Chile, se consideraron las siguientes variables: tipo de investigación, orientación epistemológica, disciplina del autor principal, plano ritzeano en sus dos dimensiones (eje $X$ naturaleza del objeto: subjetivo-objetivo; eje $Y$ nivel de análisis del objeto de estudio: micro-macro). Con dicha finalidad se realizó un análisis de correspondencias múltiples a fin de observar a nivel exploratorio las relaciones que presenta el conjunto de variables analizadas.

Tabla 5. Resumen del modelo

\begin{tabular}{|c|c|c|c|}
\hline \multirow[b]{2}{*}{ Dimensión } & \multirow{2}{*}{$\begin{array}{c}\text { Alfa de } \\
\text { Cronbach }\end{array}$} & \multicolumn{2}{|c|}{ Varianza Explicada } \\
\hline & & $\begin{array}{c}\text { Total } \\
\text { (autovalor) }\end{array}$ & Inercia \\
\hline 1 & ,748 & 2,488 & ,498 \\
\hline 2 & 601 & 1,926 & ,385 \\
\hline Total & & 4,414 & 883 \\
\hline Media & ,684(a) & 2,207 & ,441 \\
\hline
\end{tabular}

(a) La media de alfa de Cronbach se basa en la media de autovalor.

Como se observa en la tabla 5, la proporción total de varianza explicada por las dimensiones incluidas en el modelo es del $88,3 \%$, en donde la primera dimensión explica el $49,8 \%$; en cambio la segunda explica el $38,5 \%$. Si dividimos la inercia total por cada una de las dimensiones, obtenemos el porcentaje total de variabilidad que explica cada dimensión al 88,3\%. De este modo, la primera dimensión presenta un $56,4 \%(0,498 / 0,883)$ de la inercia total, en cambio la segunda dimensión presenta $43,6 \%(0,385 / 0,883)$. 
Tabla 6. Medidas de discriminación por variable

\begin{tabular}{|l|c|c|c|}
\hline \multirow{2}{*}{} & \multicolumn{2}{|c|}{ Dimensión } & \multirow{2}{*}{ Media } \\
\cline { 2 - 3 } & 1 & 2 & \\
\hline Objeto &, 319 &, 111 &, 215 \\
\hline Naturaleza &, 034 &, 318 &, 176 \\
\hline Disciplina Autor principal &, 603 &, 771 &, 687 \\
\hline Epistemología &, 708 &, 079 &, 393 \\
\hline Tipo Investigación &, 823 &, 647 &, 735 \\
\hline Total activo & 2,488 & 1,926 & 2,207 \\
\hline
\end{tabular}

En la tabla 6 se observan las medidas de discriminación por variables en cada dimensión, en donde "tipo de investigación", "epistemología" y "objeto" son tres variables que están mayormente relacionadas con la primera dimensión (naturaleza del fenómeno, continuo objetivo-subjetivo), en cambio naturaleza y disciplina del autor principal se vinculan con la segunda dimensión (nivel de análisis del estudio, continuo micro-macro). Los atributos de las variables antes indicadas en el eje 1 estarían vinculadas a investigaciones de naturaleza objetiva (en el modelo de Ritzer a una perspectiva epistemológica empírico-analítica de investigaciones evaluativas y causales), junto con las disciplinas de los autores principales provenientes de la economía y terapia ocupacional. En cambio en los estudios vinculados a naturaleza subjetiva, encontramos investigaciones de corte descriptivo, correlacional y teóricas, las disciplinas vinculadas son la enfermería, sociología, antropología, agricultura y psiquiatría (y el programa epistemológico hermenéutico). En cambio en el eje 2, vinculado a la dimensión que plantea Ritzer del nivel de análisis de los estudios (micro-macro), encontramos que en su nivel micro se posicionan los objetos intersubjetivos asociados a investigaciones evaluativas y teóricas; a las disciplinas de la sociología, terapia ocupacional y bajo un enfoque epistémico hermenéutico. En cambio en el nivel macro se encuentran estudios de naturaleza objetiva, vinculados a estudios descriptivos, correlacionales y causales; a disciplinas de la psiquiatría, psicología, antropología y agricultura; junto a la corriente epistémica empírico-analítica.

\section{Conclusiones}

El presente artículo dio cuenta del estado de las publicaciones existentes en Chile respecto al estudio del bienestar subjetivo, campo que se ha venido desarrollando a partir del año 2002 y principalmente cultivado desde la psicología. Por el nivel de publicaciones, se puede considerar esta área de estudio en un estado emergente, con un índice de colaboración promedio que no se diferencia de otros campos disciplinarios. Se evidenció que la calidad de sus publicaciones se encuentra en las de mayor prestigio dentro del contexto nacional e internacional, en tanto, se publican sus resultados en revistas indexadas en bases de datos Thompson Reuters, SciELO y Scopus. El marco epistémico de los artículos se concentran bajo el enfoque empírico analítico, de naturaleza cuantitativa y profundidad correlacional. De acuerdo a los criterios planteados por Ritzer, se evidenció que las publicaciones se concentran en los niveles macro-meso social, de orden subjetivo y en estudio de orden temporal transversal.

En relación al análisis factorial fue posible representar los elementos del plano ritzeano tanto en sus dimensiones de la naturaleza del objeto (subjetivo-objetivo) y de su nivel de análisis (micro-macro). De igual modo, nos permitió reconocer asociaciones entre los atributos de las variables analizadas. 
A partir de los resultados expuestos, es posible inferir la necesidad de avanzar en estudios longitudinales tendientes a medir las variaciones en los niveles de bienestar subjetivo a partir de eventos personales como sociales, así como en investigaciones de profundidad predictiva, involucrando factores de naturaleza tanto subjetivos como objetivos. De igual modo, dichos resultados muestran el déficit en estudios de naturaleza cualitativa tendientes a identificar los sentidos o significados atribuidos al bienestar subjetivo, así como a contrastar la hipótesis que dichos significados son compartidos cuando se aplican escalas para su medición.

\section{Agradecimientos}

Esta publicación es producto del patrocinio de la Dirección de Investigación de la Universidad del Bío-Bío, proyecto regular $N^{\circ} 150524$ 4/R.

\section{Bibliografía}

Cea, M. 2001. Metodología cuantitativa: estrategias y técnicas de investigación social. Barcelona: Síntesis.

Diener, E; Suh, E; Lucas, R. y Smith, H. 1999. Subjective well-being: three decades of progress. Psychological Bulletin 125(2): 276-302. doi: 10.1037/0033-2909.125.2.276

Martikainen, P; Bartley, M. y Lahelma, E. 2002. Psychosocial determinants of health in social epidemiology. International Journal of Epidemiology 31(6): 1091-1093. doi:10.1093/ije/31.6.1091

Pinquart, M. y Silbereisen, R. 2004. Human development in times of social change. International Journal of Behavioral Development 28(4): 289-298. doi: 10.1080/01650250344000406

PNUD. 2012. Bienestar subjetivo: el desafío de repensar el desarrollo. Santiago: PNUD Ediciones.

Ritzer, G. 1997. Teoría sociológica contemporánea. México: McGraw-Hill.

Ryff, C. 1989. Happiness is everything, or is it? Explorations on the meaning of psychological well-being. Journal of Personality and Social Psychology 57(6): 1069-1081. doi: 10.1037/0022-3514.57.6.1069

Ryff, C. y Keyes, C. 1995. The structure of psychological well-being revisited. Journal of Personality and Social Psychology 69(4): 719-727. doi: 10.1037/0022-3514.69.4.719

Sancho, R. 1990. Indicadores bibliométricos utilizados en la evaluación de la ciencia y la tecnología. Revista Española de Documentación Científica 13(3-4): 842-865.

Recibido el 29 Jul 2015

Aceptado el 5 Sep 2015 